\title{
Análise das Plataformas de Desenvolvimento Mobile aplicados na Área Educacional, usando Android e Windows Phone. Estudo de Caso: Aplicativo Planetas no Windows Phone
}

\author{
Terumi P. B. Kamada. CEDSMI ${ }^{1}$ - Bioinformática - UFPR terumikamada@live.com \\ Jayson Carpejani. CEDSMI ${ }^{1}$ - UFPR carpejani@msn.com \\ Celso Yoshikazu Ishida. CEDSMI ${ }^{1}$ - UFPR cedsmi@ufpr.br \\ Márcio Luiz Rossato Gomes. MIC ${ }^{2}$ - marciog@ techresult.com.br \\ Luiz Antônio Pereira Neves. Bioinformática - UFPR neves@ufpr.br
}

Esta pesquisa exibe uma análise comparativa de duas plataformas de desenvolvimento de tecnologias móveis: Android SDK para Eclipse IDE (Android) e o Windows Phone SDK para Visual Studio IDE (WP) e como a concepção de aplicativos móveis podem ser utilizados na área da educação. A metodologia é uma abordagem empírica através de comparativo das plataformas, os procedimentos são observacionais de acordo com a disponibilidade de funções para realização de um aplicativo e do nível de dificuldade de execução dos comandos de manipulação, que o desenvolvedor de software pode encontrar. Os resultados desta pesquisa mostram que o Windows Phone apresenta o melhor desempenho no Ambiente de Desenvolvimento Integrado, sendo a ferramenta mais eficiente para criação visual da interface do aplicativo.

Palavras-chave: Android, Eclipse, Plataforma de Desenvolvimento, Tecnologia Móvel, Windows Phone.

\begin{abstract}
This research presents a comparative analysis between two developments of Mobile Platforms: Android SDK for Eclipse IDE (Android) and Windows Phone SDK for Visual Studio IDE (WP). In addition, a sample of an application that can be used in Basic Education. The methodology is an empirical approach through a comparison of the platforms, using observational procedures. These are in accordance with the availability of functions to perform an application and based on the level of difficulty of execution of manipulation commands where the developer can find during the development. These results have shown that Windows Phone presents the best performance in the integrated development environment and thus being the most efficient tool for visual creation of the application interface.
\end{abstract}

Keywords: Android, Eclipse, Development Platform, Mobile Technology, Windows Phone.

\section{Introdução}

Aplicativos móveis constituem um software de serviço, entretenimento e comunicação específicos para dispositivos móveis com navegadores e acesso à Internet em sites com tecnologia e características mobile. A disponibilização de recursos para criar programas por meio de novas tecnologias apresenta a forma principal da concepção de um aplicativo para

$\mathrm{v}^{1}$ CEDSMI - Curso de Especialização em Desenvolvimento de Software em Mercados Internacionais

${ }^{2} \mathrm{MIC}$ - Microsoft Innovation Center 
celular, o desenvolvedor pode obter orientações elementares para baixar as ferramentas gratuitas da Internet, instalar e configurar o ambiente de desenvolvimento, criar aplicativos e disponibilizá-los nas lojas

irtuais. Entretanto, estudos apropriados, que suportem aplicações para a área educacional, ainda não foram totalmente desenvolvidos de forma integrada tais como: metodologias de ensino móvel (Mobile learning ou M-Learning) (Ozdamli e Cavux, 2011) e formas pedagógicas de desenvolvimento de aplicativos utilizados para este fim. Sendo assim, este trabalho tem como objetivo principal oferecer uma contribuição no nível educacional à comunidade, fornecendo um estudo em dois diferentes modelos de Plataformas de Desenvolvimento em tecnologias móveis: Android e Windows Phone. Estas ferramentas são as mais utilizadas atualmente, que podem ser usadas na elaboração de aplicativos educacionais.

As funções disponíveis dos celulares ou smartphones aos usuários incluem conversação, convergência, portabilidade, entretenimento, personalização, conexão múltiplas redes, além de produção de informação (Lemos, 2007), características que podem ajudar na disseminação do conhecimento e que atuam como particularidades sociais e de integração, fazendo parte da inovação tecnológica (Valentim, 2009; Ferneley e Light, 2008), inclusive auxiliando o sistema educativo e facilitando o acesso à informação em programas de ensino (Gartner, 2012; Pelissoli e Loyolla, 2004). O interessante é que a interatividade, proporcionada pela facilidade de acesso pelo usuário às funcionalidades avançadas destas ferramentas, tem notoriedade na ação dos planos tecnológicos dos governos. Um exemplo da aplicação prática de aplicativos mobiles é dado pela prefeitura cidade de São Francisco (US) que incentiva seus cidadãos a desenvolverem sistemas que podem ajudar na melhoria de uma cidade, tais como: aplicativos que disponibilizem indicativos de serviços de reciclagem, dados de áreas violentas, meios e fluxos de transportes e até mesmo, o controle ambiental da cidade, como por exemplo: o tipo de árvores existentes na cidade e suas manutenções (DATASF, 2012).

Dentre outras aplicações, os dispositivos móveis são utilizados como meio de comunicação digital que pode influenciar famílias na forma de educar seus filhos (Chiong e Shuler, 2010). Neste contexto, a análise comparativa das plataformas de desenvolvimento pode ajudar a desenvolver e implantar dispositivos móveis inteligentes, que em curto prazo, é uma opção para ajudar a promover e transformar a educação das crianças. Fica evidente que a nova geração de dispositivos móveis pode desempenhar na aprendizagem dos seres humanos, um papel notório, eles podem aprender a partir da adoção de dispositivos e serviços mobile (Valentin, 2009). Atualmente, percebe-se que a maioria das crianças é capaz de manusear um dispositivo móvel por conta própria, sem maiores dificuldades, de forma independente. Portanto, o uso de dispositivos móveis como ferramenta complementar à educação é uma oportunidade para incentivar, em um contexto de mundo real, e fornecendo uma ponte entre a escola e a casa do aluno, a colaboração, autoaprendizagem e as interações sociais de forma positiva (Millstone, 2012).

Outro ponto interessante, além da integração com outras tecnologias e da inclusão social entre vários grupos de utilizadores que os dispositivos móveis proporcionam (Ferneley e Light, 2008), é o custo relativamente baixo e acessibilidade facilitada às comunidades de baixa renda, se comparado com outras tecnologias, principalmente em países em desenvolvimento. Com as vendas de smartphones no mundo atingindo cerca de 468 milhões de unidades em 2011, há aumento de 57,7 \% a partir de 2010, e estimativas de 
superação da marca de 1 bilhão de aparelhos, em $47 \%$ do mercado total de dispositivos móveis, até 2015. O setor está fortalecendo no mercado e acarreta a oportunidade de fabricação de smartphones de baixo custo, sobretudo em mercados emergentes (Gartner, 2012). Neste sentido, a acessibilidade está sendo bem explorada. Por exemplo, no Google Play (Android, 2012a), segmento de mercado para Android, onde os desenvolvedores inserem suas aplicações e os usuários podem obter uma cópia para o dispositivo (baixar), cerca de $50 \%$ dos aplicativos são gratuitos e os pagos tem um valor baixo (Android, 2012a).

O mercado de aplicativos cresce rapidamente, em 05 de março de 2012, a Apple confirmou que em sua loja "App Store" (Apple, 2012), lançada a menos de quatro anos, houve mais de 25 bilhões de "downloads" de seus aplicativos. A loja "App Store" já pagou mais de 4 bilhões de dólares para os desenvolvedores (Apple, 2012). As lojas virtuais oferecem aplicativos para usuários em vários países ao redor do mundo, encontrados em categorias incluindo jogos, negócios, saúde e educação. Seu concorrente Windows Phone mostra uma aceleração na taxa de aplicativos publicados, uma média de 265 novos itens foi adicionada ao "Marketplace" a cada dia, nas 17 categorias existentes, (Marketplace, 2012). O mercado chegou a 40.000 itens em 16 de novembro de 2011, o que significa a taxa de adição está se aumentando. As causas prováveis são em primeiro lugar a disponibilidade de expansão do mercado que geograficamente foi ampliado de 16 para 35 países e a parceria com dispositivos da Nokia (Windows Phone, 2011), mostra a amplitude que o mercado poderá atingir.

Os aplicativos educacionais para crianças ainda é um mercado pouco explorado, apesar dos altos investimentos do mercado, somente $2 \%$ dos aplicativos são enquadrados na área de Educação, apresentando uma oportunidade para os produtos de alta qualidade, que podem ser divertidos e ao mesmo tempo educativos. Shuler (2009) afirma que $47 \%$ dos 100 melhores aplicativos vendidos têm foco na educação de crianças, assim o mercado para aplicativos educativos para crianças deve ser considerado um passo importante. Investimentos em lojas como Marketplace do Windows Phone (Figura 1) facilitam a distribuição de aplicativos, onde 50\% são baixados gratuitamente (Windows Phone, 2011). 


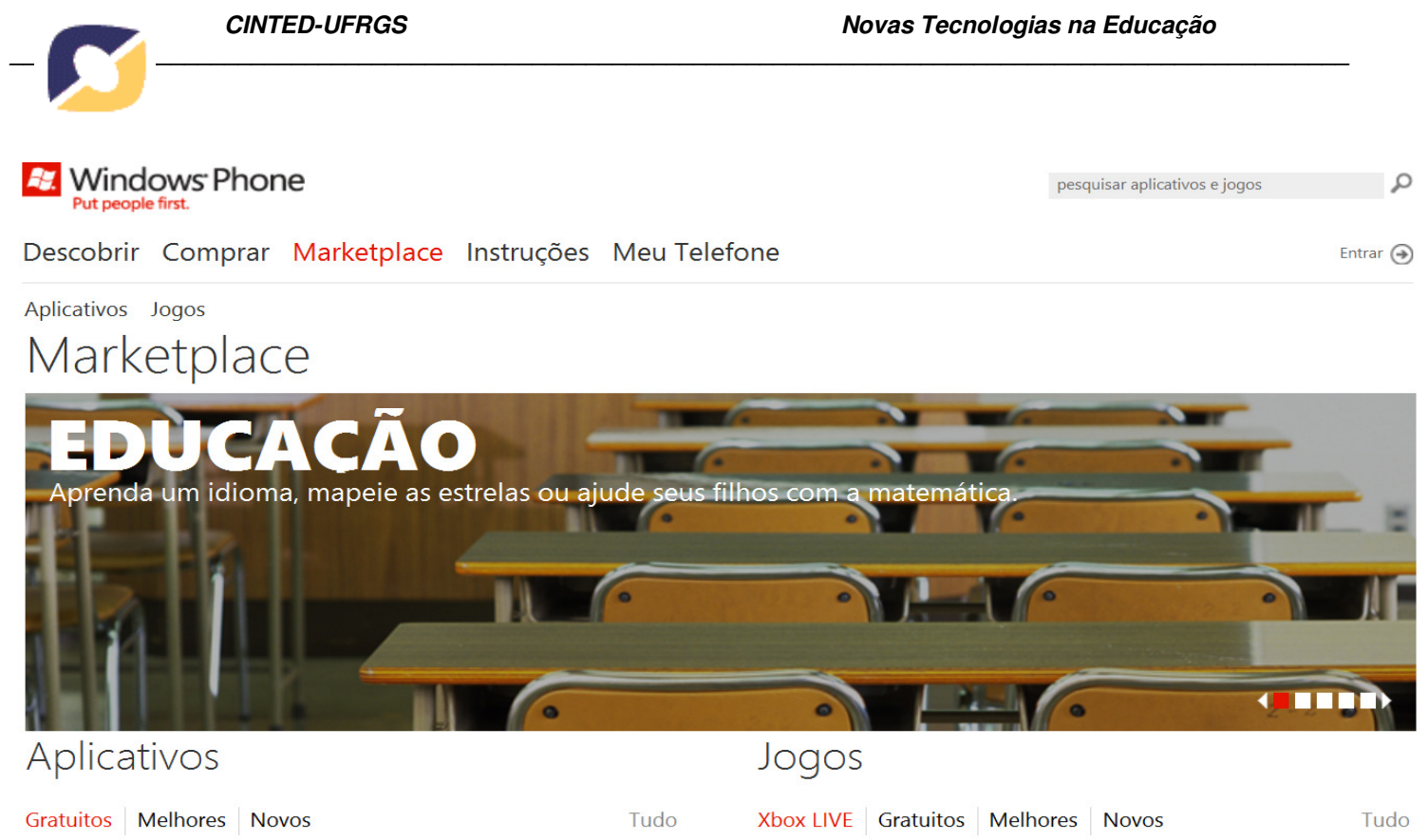

Figura 1. Marketplace Windows Phone. Catura da imagem Marketplace, loja Windows Phone com Aplicativos e Jogos disponíveis para área da Educação

Os desenvolvedores podem, por meio de ferramentas que facilitam a concepção dos sistemas mobile, especificar, projetar, implementar e comercializar suas ideias. Preece (1994) afirma que já nos primeiros estágios de criação de um software, principalmente na fase de design, as ponderações dos desenvolvedores em relação à criação dos seus sistemas são feitas observando-se a possibilidade de utilização do produto final comercialmente, as lojas virtuais, abordadas anteriormente, exibem a facilidade de comercialização dos aplicativos. O processo de criação pelos desenvolvedores utilizando as Plataformas de Desenvolvimento Mobile é interativo e fácil, adapta os modelos de softwares com as características de interface e da funcionalidade de cada aplicação, principalmente quando utiliza o emulador, recurso disponibilizado pelas plataformas que reproduz as funções de um ambiente determinado, o que contribui para testar novas ideias de maneira rápida e informal. A descrição e a comparação das Plataformas Android e Windows Phone, nas seções seguintes, exibem características específicas de cada tipo de ferramenta e serve como base para a escolha da produção e comercialização do aplicativo.

\section{Material e Métodos}

A metodologia definida está organizada em duas etapas a fim de atingir os objetivos propostos nesta pesquisa, usando uma abordagem empírica. A primeira etapa é a descrição e identificação das duas principais plataformas associadas ao campo de desenvolvimento mobile Android SDK e Windows Phone SDK, contextualizando-as dentro do campo da prática do desenvolvedor, em particular descrevendo os recursos dos principais dispositivos e técnicas das ferramentas, bem como os propósitos pedagógicos a que podem servir ou adotar. A segunda etapa é a análise da comparação das características das ferramentas, examinando criticamente as suas possibilidades e sistematizando modelos e propostas de cada tipo de desenvolvimento. Esta última etapa tem procedimento observacional com o estudo de desempenho entre as ferramentas, usando procedimento comparativo. A terceira 


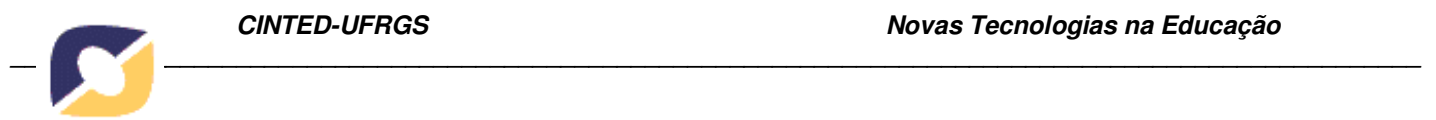

etapa exibe os Resultados e Discussões contendo um estudo de caso de aplicativo desenvolvido para área de educação básica. A última etapa mostra as conclusões obtidas neste artigo.

\subsection{Descrição das Plataformas para Desenvolvimento Mobile}

Para realizar a caracterização das ferramentas de desenvolvimento Mobile foram definidos, de acordo com o mercado, dois modelos de ferramentas para desenvolvimento distinto, o Android SDK, como sistema operacional móvel principal e seu concorrente, o Windows Phone SDK, mostra investimento na capacidade produtiva do desenvolvedor com taxa ascensão positiva no número de aplicativos. Os SDKs (Kits Desenvolvimento Software) destas plataformas são disponibilizados pelas organizações para que os programadores externos tenham uma melhor integração com o sistema proposto, pode incluir documentação, código e utilitários. Os programadores desenvolvem suas aplicações de acordo com um padrão de desenvolvimento para os sistemas operativos em questão.

\subsubsection{Windows Phone SDK}

Plataforma SDK, utilizada por desenvolvedores.NET (MSDN, 2012c), é uma ferramenta que permite a criação de aplicativos utilizando as tecnologias Microsoft. Ela é utilizada para projetar e criar aplicativos para Windows Phone. Esta ferramenta gera código para Framework.NET e suporta linguagens Visual Basic .NET, C\#, C++, e J\#. Trabalha com tecnologias como Visual Studio, Silverlight e .NET, WPF, Silverlight e XAML. Como processo de melhoria da plataforma, em 2011, surge a versão com o codinome "Mango", com mais de 500 recursos, destaque de melhoramento como o suporte a multitarefa, atualização da versão do Silverlight para o Silverlight 4 e atualização do Browser para a versão do Internet Explorer 9 com suporte ao HTML5, mudanças tanto para o desenvolvedor como para o usuário. As melhorias incluem uma melhor integração com o Skydrive, com as funções de compartilhamento de fotos e de vídeos, com "Upload" em background, para o arquivamento nas nuvens. Além de realizar pesquisas de conteúdo, como um dos recursos de integração. Com as ferramentas disponíveis, pode-se criar e testar o aplicativo com a ferramenta Visual Studio 2010 e utilizar funções do emulador, sistema que está integrado com o Expression Blend4 para o Windows Phone (Figura 2). A integração com o Blend4 possibilita a criação visual da interface com produtividade (Kamada, 2012).

O desenvolvimento do código em XAML pelo Expression Blend também pode ser reproduzido no Visual Studio 2010 utilizando o IDE - Integrated Development Environment ou Ambiente de Desenvolvimento Integrado. 


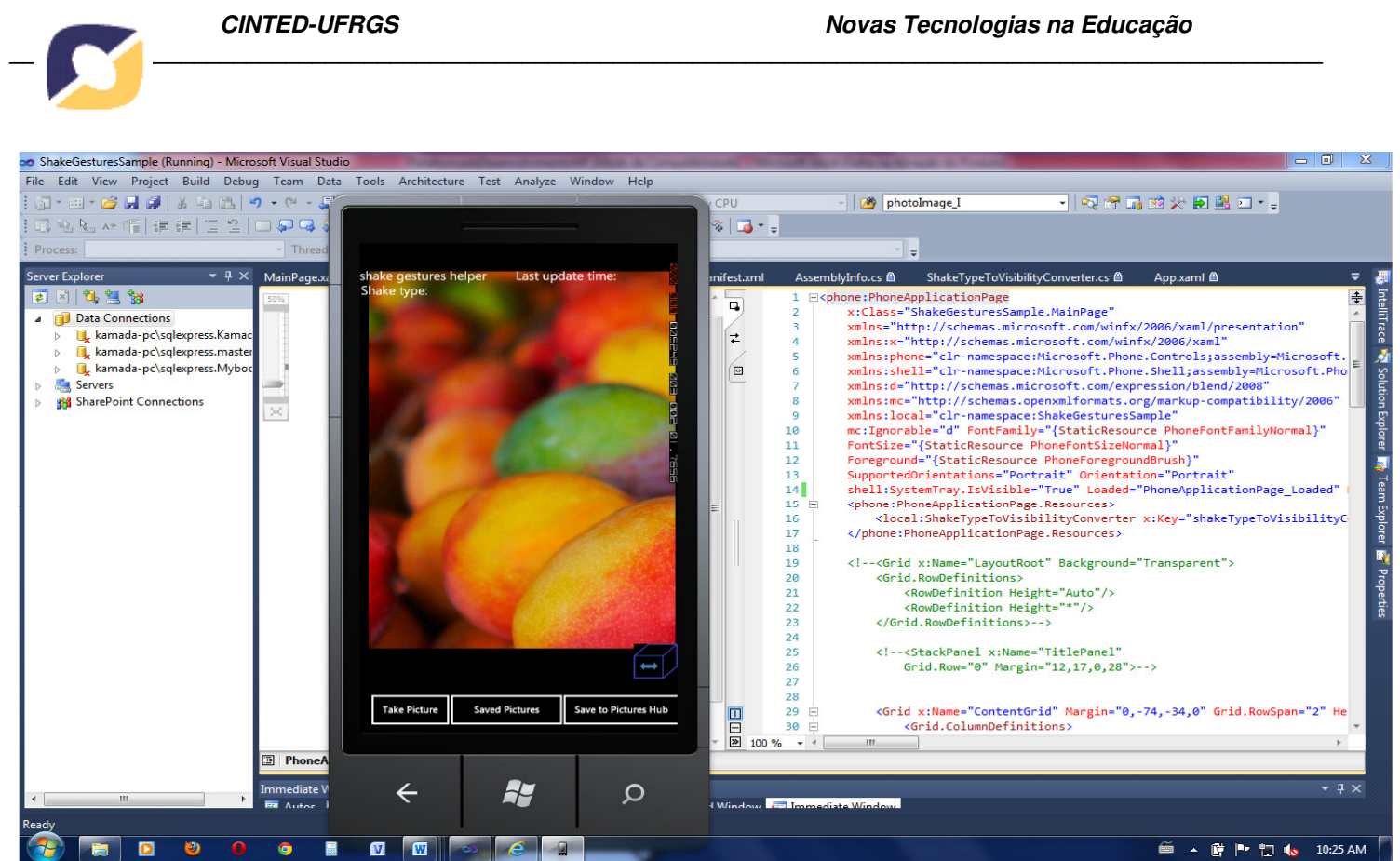

Figura 2. Ferramenta de Desenvolvimento para Mobile - Visual Studio (Kamada, 2012).

Catura da imagem Visual Studio 2010 com emulador em modo debugging (Processo de encontrar e reduzir defeitos) e visualização do design e marcação XAML. O Visual Studio, utilizando o IDE, com resposta de edição simultanea com Blend 4

\subsubsection{ANDROID SDK}

O Eclipse (IDE) é uma ferramenta código aberto que agrega à plataforma de desenvolvimento os geradores de códigos Java, e por meio de extensões origina códigos em Python e $\mathrm{C} / \mathrm{C}++$. O Eclipse começou como tecnologia proprietária com o lançamento entre 1999 e 2001 da versão 1.0, anunciado pela empresa americana IBM (International Business Machines), no valor de US\$ 40 milhões (Android, 2012).

O objetivo da IBM era reduzir o número de ambientes de desenvolvimento incompatíveis, aumentando a reutilização dos componentes comuns nesses ambientes. A estrutura comum equipes de desenvolvimento podem, além de aproveitar componentes, permitir que os desenvolvedores integrem-se entre os projetos. (Eclipse, 2012). O Android, usado como módulo de extensão (Plug-in) foi incorporado à plataforma Eclipse e teve sua origem em um grupo de empresas o Open Handset Alliance (OHA) que é uma aliança de diversas empresas com a intenção de criar padrões abertos para telefonia móvel, em julho de 2005. As bibliotecas do Android SDK são compatíveis com os ambientes de desenvolvimento para Eclipse (Figura 3), JDK (Kit Desenvolvimento Java), Android, Ferramenta de Desenvolvimento Plug-in (Módulo de extensão) e Apache (Android, 2012b). 


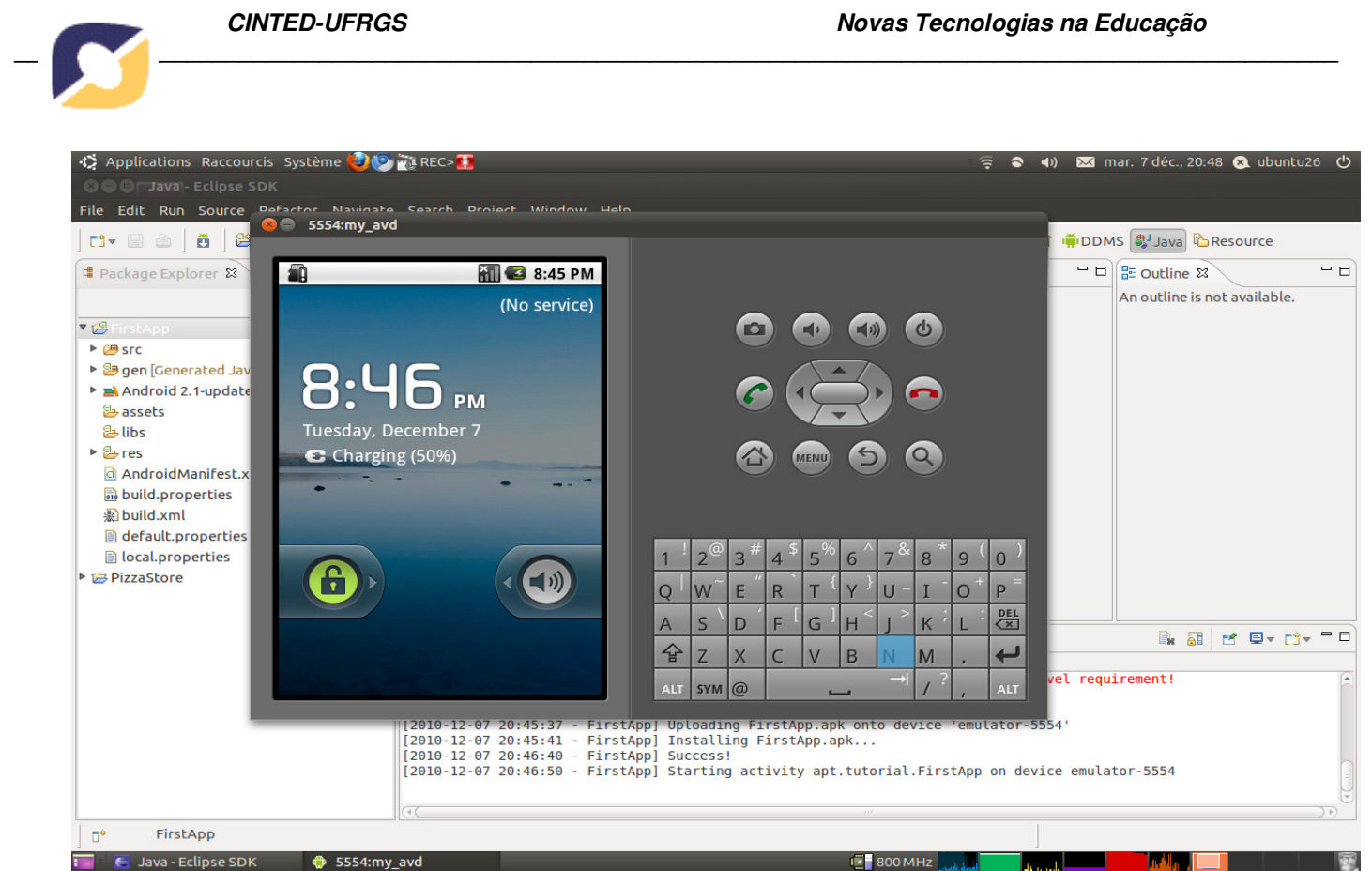

Figura 3. Android Integrado Eclipse. Captura da imagem do Eclipse com exemplo do emulador em modo debugging (Processo de encontrar e reduzir defeitos), permite a visualização e simulação de teclado o mesmo tempo. Observa-se o a compilação JAVA

\subsection{Análise da comparação das características das ferramentas.}

Como estratégia de levantamento das ferramentas foi realizada uma revisão com base nas características entre Windows Phone SDK e Android SDK, a Tabela 1 exibe as diferenças técnicas encontradas.

Tabela 1. Características das Ferramentas Android e Windows Phone baseada nos dados oficiais Android (2012b) e MSDN (2012a)

\begin{tabular}{|c|c|c|}
\hline & ANDROID & WINDOWS PHONE \\
\hline Características & $\begin{array}{l}\text { Com o lançamento do SDK, características e } \\
\text { especificações facilmente distribuídas. }\end{array}$ & $\begin{array}{l}\text { Baseado no Windows CE } 6.0 \text {, o que significa que possui um } \\
\text { kernel de } 32 \text { bits, suporte máximo de } 4 \text { GB de RAM. }\end{array}$ \\
\hline Handset Layouts & $\begin{array}{l}\text { A plataforma é adaptada tanto para } \\
\text { dispositivos VGA maiores, gráficos 2D, } \\
\text { bibliotecas gráficas 3D baseadas em OpenGL } \\
\text { ES especificação } 2.0 \text { e layouts tradicionais. }\end{array}$ & $\begin{array}{l}\text { Pré-Requisito: Windows Live ID. Interação: Três redes sem } \\
\text { mudar de localização. SMS, Chat do Facebook e Live } \\
\text { Messenger com mesma tela de conversa. }\end{array}$ \\
\hline Armazenamento & É utilizado SQLite para armazenamento de dados. & $\begin{array}{l}\text { SQL CE, Página Estado - Estado do aplicativo, } \\
\text { armazenamento isolado. }\end{array}$ \\
\hline Mensagens & $\begin{array}{l}\text { Disponibilidade de envio de } \\
\text { mensagens: SMS e MMS. }\end{array}$ & $\begin{array}{l}\text { O Windows Phone combina as mensagens do utilizador em } \\
\text { "conversas", que permitem ao utilizador contatos por meio do } \\
\text { Windows Live Messenger e chat do Facebook. }\end{array}$ \\
\hline Navegador & $\begin{array}{l}\text { Baseado no framework de Código aberto - } \\
\text { WebKit. }\end{array}$ & $\begin{array}{l}\text { Embutido versão 'Internet Explorer Mobile' com um motor } \\
\text { de renderização baseado no Internet Explorer } 9 .\end{array}$ \\
\hline $\begin{array}{l}\text { Máquina Virtual } \\
\text { Dalvik }\end{array}$ & $\begin{array}{l}\text { Aplicações em Java compiladas em bytecodes e } \\
\text { executadas Máquina virtual Dalvik, especializada } \\
\text { para uso em dispositivos móveis. Os programas } \\
\text { podem ser distribuídos em formato binário e } \\
\text { executados em qualquer dispositivo Android, } \\
\text { independentemente do processador. Apesar das } \\
\text { aplicações serem escritas na linguagem Java ela } \\
\text { não é uma máquina virtual Java, já que não } \\
\text { executa bytecode JVM. }\end{array}$ & $\begin{array}{l}\text { O Windows Phone tem acesso incorporado ao SkyDrive a } \\
\text { partir dos centros Office e Imagens. Programas para edição: } \\
\text { Word, Excel, PowerPoint, OneNote Mobile, SharePoint } \\
\text { Workspace Mobile. }\end{array}$ \\
\hline Multimídia & $\begin{array}{l}\text { O sistema disponibiliza formatos de áudio e } \\
\text { vídeo: MPEG-4, H.264, MP3, e AAC. }\end{array}$ & $\begin{array}{l}\text { O sistema disponibiliza formatos de áudio e vídeo: MPEG- } \\
\text { 4, H.264, MP3 }\end{array}$ \\
\hline
\end{tabular}




\begin{tabular}{|c|c|c|}
\hline \multicolumn{2}{|r|}{ CINTED-UFRGS } & Novas Tecnologias na Educação \\
\hline $\begin{array}{l}\text { Suporte Adicional } \\
\text { de Hardware }\end{array}$ & $\begin{array}{l}\text { Capaz de fazer uso de câmeras de vídeo, tela } \\
\text { sensível ao toque, GPS, acelerômetros, e } \\
\text { aceleração de gráficos 3D. }\end{array}$ & $\begin{array}{l}\text { Conjunto de botões de hardware que incluem o botão Iniciar, } \\
\text { Pesquisar e Voltar. Tela com resolução WVGA ( } 480 \text { X } 800 \\
\text { pixels) capaz de renderizar conteúdo da web a largura da } \\
\text { página inteira e exibir filmes em widescreen. Capacitiva } \\
\text { multitouch com no mínimo } 4 \text { pontos. Suporte para } \\
\text { conectividades de dados utilizando as redes de celular (GSM, } \\
3 G \text {, etc) e Wi-Fi,256Mb de memória RAM, } 8 \text { Gb de memória } \\
\text { interna, A-GPS, Acelerômetro, Bússola, Giroscópio, Câmera } \\
\text { primária Câmera secundária (frontal). }\end{array}$ \\
\hline $\begin{array}{l}\text { Ambiente de } \\
\text { Desenvolvimento } \\
\text { (SDK) }\end{array}$ & $\begin{array}{l}\text { Inclui um emulador, ferramentas para debugging, } \\
\text { memória e análise de desempenho. O Eclipse } \\
\text { (IDE) (atualmente } 3.4 \text { ou 3.5) poderá ser utilizado } \\
\text { através do plugin Android Develpment Tools } \\
\text { (ADT). }\end{array}$ & $\begin{array}{l}\text { Visual Studio ambiente de desenvolvimento integrado (IDE). } \\
\text { Microsoft Advertising SDK Windows Phone, Kit possui dois } \\
\text { emuladores, Microsoft Visual Studio 2010 Express para } \\
\text { Windows Phone-Emulador Windows telefone-Windows } \\
\text { Phone SDK 7.1. Assembléias· Silverlight } 4 \text { SDK e } \\
\text { DRT.Windows Phone SDK 7.1. Extensões para o XNA } \\
\text { Game Studio 4.0. Microsoft Expression Blend. SDK para } \\
\text { Windows Phone 7.1. Expression Blend. SDK Windows } \\
\text { Phone OS 7.1. WCF Data Services }\end{array}$ \\
\hline
\end{tabular}

\section{Resultados e Discussão}

A tecnologia é um fator de mudança social (Valentim, 2009), inclusive na educação, porém o acesso diferenciado à tecnologia e à diversidade entre os dispositivos móveis representa um desafio para professores e alunos que desejam utilizar estes recursos para melhorar seus resultados acadêmicos, assim como para os fabricantes que buscam facilitar essa aprendizagem. Uma das principais desvantagens no uso de aplicativos móveis na área educacional inclui o potencial de distração da criança, preocupações com a saúde e questões de privacidade de dados. Ferramentas como SMS e ligação são dispositivos para a maioria dos usuários, mas experiências através de GPS, Realidade aumentada, QR, Codes, Aplicativos Mobile, Bluetooth ainda apresentam um acesso limitado em países como o Brasil.

A facilidade em trocar informação com outros dispositivos e o acesso à internet torna o aprendizado por meio dos dispositivos móveis de alta usabilidade (Pelissoli e Loyolla, 2004), um exemplo é o uso de informação geográfica relacionada a itens multimédia, como nas fotografias JPG (Valentim, 2009).

A análise da comparação das características das ferramentas mostrou que o Android SDK tem uma desvantagem no desenvolvimento de interface, sendo pouco intuitivo. Os vários tamanhos de tela, com proporções da altura e largura diferentes, prejudica a produtividade dos programadores. Tem como vantagens um sistema operacional aberto que funciona de forma leve junto aos aparelhos com menos hardware, possui tela mais sensível ao toque em análise a outros aparelhos antigos. Possui aplicativos grátis no Google Play, conexão com as redes sociais, conexão com a internet e aparelhos mais baratos nas operadoras. As desvantagens incluem aplicativos pagos mais caros que em outras lojas, o que pode prejudicar o público específico da área da educação, falta de atualização e da análise dos aplicativos do sistema operacional para os aparelhos, que gera diferenças nas funcionalidades dos aplicativos entre alguns aparelhos.

Segundo esta análise é observado que o Windows Phone tem a vantagem de possuir Microsoft Office Mobile, ferramenta já instalada no telefone, com versões embutidas do Word, Excel e PowerPoint, que são programas de uso comum para edição e formatação de documentos tabelas, marcadores, imagens, planilhas, gráficos e fórmulas. Estas funcionalidades operam de modo esperado pelo usuário. As principais ressalvas impostas 
pela Microsoft são feitas aos fabricantes de smartphones principalmente em relação ao hardware, pois não é projetado para rodar com eficiência sobre circuito integrado em um chip, com dois núcleos de CPU. A Microsoft também estabelecia especificações de processadores que rodem com pelo menos a $1 \mathrm{GHz}$ e câmeras de 5 MP (MSDN, 2012b).

Um dos itens que mais chama a atenção na plataforma da Microsoft é a facilidade na instalação da SDK do Windows Phone, pois todos os componentes para o desenvolvimento são instalados usando apenas um único formulário de instalação. Devido a isto, destaca-se, nesta plataforma, como diferencial, a disponibilidade do item Panorama ao desenvolver na construção dos projetos.

Foi encontrada, no Android SDK, uma maior portabilidade entre vários celulares, abrangendo um número maior de dispositivos móveis, porém observou-se uma dificuldade da adaptação de tamanho de tela entre os diversos aparelhos, por parte dos desenvolvedores. Na comparação da plataforma nota-se que o SDK do Android exige uma maior experiência do desenvolvedor com a necessidade de conhecimento da melhor adoção dos dispositivos. No SDK Windows Phone a maturidade pode ser menor, pois todas as ferramentas auxiliares estão integradas ao IDE Visual Studio, não existe a necessidade de um conhecimento prévio de qual dispositivo móvel será desenvolvido.

\subsection{ESTUDO DE CASO}

A adoção de smartphone proporcionou o uso do aparelho como ferramenta didática. Nesta seção é apresentado um aplicativo educacional para Windows Phone (Figura 4), desenvolvido na plataforma móvel da Microsoft. O Aplicativo tem o objetivo de agir como instrumento de comunicação imediata aos estudantes, provocando a colaboração entre os alunos e assim, criando um ambiente colaborativo dentro das salas de aulas. Planetas é um aplicativo sobre o sistema solar, com a utilização de uma linguagem autoexplicativa, que faz parte de uma série chamada Cienciatorium. Esta série foi desenvolvida por um dos autores deste artigo e engloba, na área de Ciências, aplicativos para aprendizagem sobre o Corpo Humano, Consciência Social, Legislação no Direito de Animais, Posse Responsável de Animais Domésticos e Linguagem dos Sinais - Libras. No aplicativo Planetas, foi utilizada, de forma intuitiva, a característica técnica WP Panorama Application, componente da SDK Windows Phone, que proporciona uma vista abrangente do ambiente. Outro controle usado foi o "ListBox" com imagens, que permite que o "Scrolling", controle gráfico do usuário, mostra um texto contínuo, melhorando a área do trabalho. 

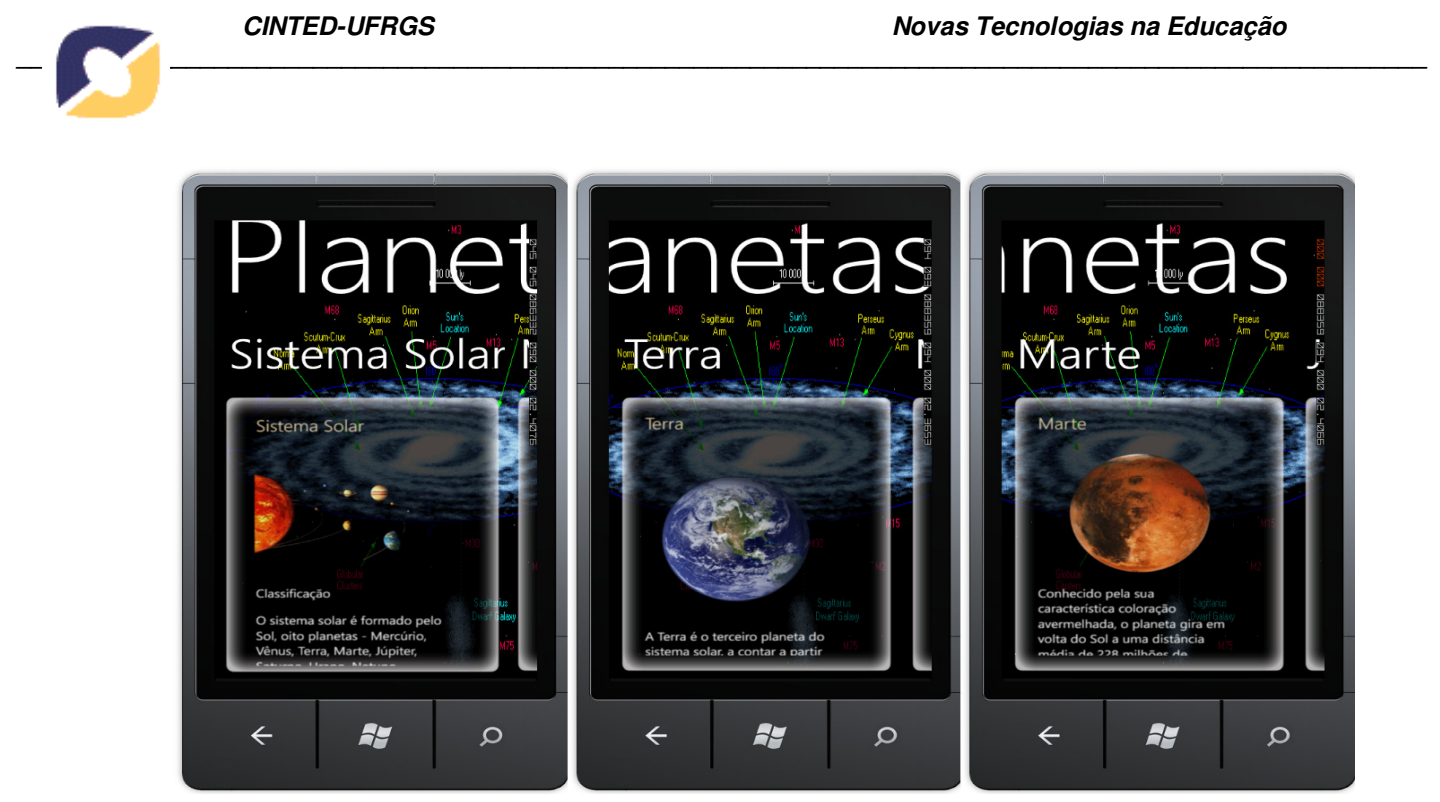

Figura 4. Aplicativo Planetas Windows Phone (Marketplace, 2012). Captura de Imagens do Aplicativo Planetas, série Cienciatorium. Com levantamento, até a data de 29/06/2012, de 1500 downloads.

A compatibilidade do aplicativo Planetas, no ambiente Microsoft, está na versão 7.5 do Windows Phone, que abrange todos os aparelhos atuais do mercado. Foi utilizado a ferramenta Expression Blend 4, utilizada para projetar e criar o design da tela (figura 4) sem precisar criar diretamente linhas de códigos, pois esta ferramenta trabalhou com interação de recursos de tecnologias do Visual Studio, WPF, Silverlight e XAML. Um dos recursos visuais desta ferramenta comporta o suporte à criação de animações, onde o acesso ao espaço de trabalho (Animation Workspace) que permite acionar animações com linhas do tempo graficamente (Timelines). O aplicativo Planetas está disponível: http://www.windowsphone.com/pt-BR/apps/67b8b46f-ed6f-49a6-aebc-021bdaae3b43. As informações são impressas na tela de forma clara e objetiva, realçando a alta interatividade dos recursos visuais e exibindo ao usuário a ideia de que ciência é informação.

\section{Conclusões}

De acordo com a metodologia proposta aplicada nesta pesquisa, os resultados obtidos em comparativos no desenvolvimento de aplicativos mostrou que o Windows Phone apresentou melhor desempenho no Ambiente de Desenvolvimento Integrado com o emulador e o BLEND 4, sendo o mais eficiente para criação visual da interface. As restrições do Sistema Operacional Android, como os vários tamanhos de tela, também chamado de fragmentação da plataforma, com proporções da altura e largura diferentes dos aparelhos, podem ser um fator importante na escolha do desenvolvedor na concepção de um aplicativo para educação. Uma das características mais interessantes do Windows Phone é a facilidade relacionada ao desenvolvimento de aplicações dentro dele, o potencial do Sistema Operacional da Microsoft é muito positivo, particularmente traz uma liberdade de desenvolvimento para os desenvolvedores do ambiente.NET, principalmente pela produtividade e pelo fato de aproveitar os conhecimentos já adquiridos em C\#, Visual Basic, XNA e Silverlight. 
Os dispositivos móveis discutidos a partir das características de cada uma das plataformas de desenvolvimento são identificados como habilitadores da realização do MLearning. O estudo realizado abordou as novas tendências em dispositivos móveis inteligentes, especificamente o efeito de explorar a viabilidade e a eficácia do uso de aplicativos para promover a aprendizagem entre crianças, para tanto os desenvolvedores devem trabalhar com faixa de usuários específicos, e desenvolver produtos que tenham em conta a presença desses espectadores em potencial. O estudo de caso do aplicativo Planetas é uma proposta interessante para a disciplina de Geografia Básica. Porém, a escassez de material comprovando a teoria da aprendizagem por meio de dispositivos móveis torna difícil uma avaliação pedagogia eficaz. Além disso, pela disponibilização de acesso à tecnologia de desenvolvimento ser excessivamente simplificada, principalmente utilizando o Windows Phone SDK, o enquadramento das tecnologias móveis no sistema de ensinoaprendizagem deve ser tratado com seriedade pelas instituições de ensino, pois, a partir dos cenários estudados percebe-se a viabilidade e a enorme potencialidade de uso dos dispositivos móveis no processo educativo.

\section{Referências}

ANDROID, Disponível em: http://source.android.com. Acesso em: 26/04/2012.

ANDROIDb, Disponível em: http://developer.android.com/sdk/adding-components.html Acesso em: 28/04/2012.

APPLE. Apple's App Store reaches 25 billion downloads. 2012. Disponível em: http://www.bgr.com/2012/03/05/apples-app-store-reaches-25-billion-downloads/. Acesso em: 24/04/2012.

CHIONG, C; SHULER, C. Learning: Is there an app for that? Investigations of young children's usage and learning with mobile devices and apps. New York: The Joan Ganz Cooney Center at Sesame Workshop. 2010. Disponível em: http://www.joanganzcooneycenter.org/Reports-27.html. Acesso em: 24/04/2012.

DATASF, Disponível em: http://datasf.org/showcase/. Acesso em: 24/04/2012.

ECLIPSE, Disponível em: http://www.eclipse.org/downloads/. Acesso em:24/04/2012.

FERNELEY, E; LIGHT, B. Unpacking user relations in an emerging ubiquitous computing environment: introducing the bystander. Journal of Information Technology, 2008.

GARTNER, Disponível em: http://www.gartner.com/it/page.jsp?id=1622614 Acesso em: 25/04/2012.

KAMADA, T.P.B. Análise de Desempenho de Ferramentas de Protipação no Desenvolvimento de Sistemas. Revista Gestão. Curitiba - PR. 2012.

LEMOS, A. Comunicação e práticas sociais no espaço urbano: as características dos Dispositivos Híbridos Móveis de Conexão Multirredes (DHMCM). Comunicação, Mídia e Consumo, Vol. 4, No 10. São Paulo, 2007.

MARKETPLACE WINDOWS PHONE. Disponível em: http://www.windowsphone.com/pt-BR/marketplace Acesso em: 10/05/2012.

MILlSTONE, J. National Survey and Video Case Studies: Teacher Attitudes about Digital Games in the Classroom. New York: The Joan Ganz Cooney Center at Sesame Workshop. 2012. Disponível em: http://www.joanganzcooneycenter.org/Reports-34.html. Acesso em: 10/05/2012. 


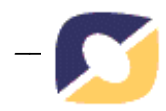

MSDNa, Disponível em: http://msdn.microsoft.com/en-us/library/ff402531(v=vs.92).aspx. Acesso em: 28/04/2012.

$\mathrm{MSDNb}$, Disponível em: http://msdn.microsoft.com/enus/library/ff637514\%28v=vs.92\%29.aspx Acesso em: 11/04/2012.

MSDNc, Disponível em: http://msdn.microsoft.com/pt-br/netframework/aa496123.

OZDAMLI, F.; CAVUS, N. Basic elements and characteristics of mobile learning, Procedia - Social and Behavioral Sciences, Volume 28, 2011, Pages 937-942, ISSN 1877-0428, 10.1016/j.sbspro.2011.11.173.

PELISSOLI, Luciano; LOYOLLA, Waldomiro. Aprendizado Móvel (M-Learning): Dispositivos e Cenários. Centro de Educação Tecnológica Prof. Luiz Rosa. Instituto UNIEMP, 2004

SHULER, C. iLearn; A Content Analysis of the iTunes App Store's Education Section, New York: The Joan Ganz Cooney Center at Sesame Workshop. 2009. VALENTIM, Hugo. Para uma Compreensão do Mobile Learning. Reflexão sobre a utilidade das tecnologias móveis na aprendizagem informal e para a construção de ambientes pessoais de aprendizagem. Gestão de Sistemas de e-Learning, Universidade Nova de Lisboa, Lisboa. 2009. Dissertação de Mestrado.

WINDOWS PHONE, All About. 2011. Acesso em: 25/04/2012. Disponível em: http: allaboutwindowsphone.com/news/item/13913_Windows_Phone_Marketplace_pass.php 